\title{
Planning and spatial working memory in Parkinson's disease
}

\author{
ROBIN G MORRIS, * JOHN JOSEPH DOWNES, " BARBARA J SAHAKIAN, $\dagger$ \\ JOHN L EVENDEN, $\ddagger$ ANDREW HEALD,§ TREVOR W ROBBINS"
}

\begin{abstract}
From the Department of Experimental Psychology, "University of Cambridge, Cambridge, Department of Psychiatry, Royal Victoria Infirmary, ${ }^{*}$ Newcastle-upon-Tyne, Section of Old Age Psychiatry, Institute of Psychiatry, $\dagger$ University of London, Merck Sharp and Dohme Neuroscience Research Centre, $\ddagger$ Harlow, Department of Neurology and Neurosurgery,§ Addenbrookes Hospital, Cambridge, UK
\end{abstract}

SUMMARY The higher level cognitive function of planning was studied in a group of medicated Parkinson's disease patients and a group of matched control subjects, using a computerised version of Shallice's Tower of London task. Baseline measurement of the ability to execute a given plan of action, to generate low level strategies required for efficient searching, and spatial working memory capacity, all of which contribute to performance on the planning task, established that the Parkinson's disease group was unimpaired on any of these measures. On the Tower of London task, the Parkinson's disease group was also unimpaired in terms of the average number of moves required to solve a problem. However, a specific planning deficit was evident when "thinking" times were analysed, and this was after the confounding influence of motor initiation and execution times had been carefully extracted from total performance times. This finding is discussed in relation to putative functions of the frontal lobes and basal ganglia, and an attention-switching hypothesis is developed to account for it.

Several studies have now established that patients with Parkinson's disease develop mild neuropsychological deficits across a range of functions, affecting such capacities as memory, visuo-spatial processing and attention. ${ }^{1-3}$ Some of these deficits are identifiable even in the early untreated stages of the disease. ${ }^{3-5}$ For a significant proportion of patients these cognitive deficits are prodromal to a state of dementia, and a conservative estimate of the increased risk of dementia associated with Parkinson's disease is $10-15 \%{ }^{6}$

Some of the deficits described in Parkinson's disease $^{7-9}$ resemble those commonly attributed to frontal lobe damage. ${ }^{10-13}$ These include impairments in fluency, concept formation, self-ordered tasks, and recency discrimination. Neuropathological research has established that there is a decrease in dopamine concentration in the prefrontal cortex asso-

\footnotetext{
Address for reprint requests: $\mathrm{Dr} \mathrm{T}$ W Robbins, Department of Experimental Psychology, University of Cambridge, Downing St, Cambridge CB2 3EB, UK.
}

Received 10 August 1987 and in revised form 12 January 1988. Accepted 21 January 1988 ciation with Parkinson's disease, ${ }^{14}$ and similar, though more substantial depletions, have been found to impair cognitive performance in primates. ${ }^{15}$ Moreover, the degeneration of noradrenergic or cholinergic cortical afferents, also associated with Parkinson's disease, ${ }^{16}$ may impair prefrontal cortex function either alone or in combination with the dopamine depletion occurring in this area.

These findings suggest a clear rationale by which frontal-type deficits might be predicted in Parkinson's disease. However, damage to the frontal areas may be sufficient, but not necessary, for producing deficits in cognition. The work of DeLong and his colleagues ${ }^{17}$ points to the existence of segregated parallel corticosubcortical loops subserving "motor" and "complex" functions. Both Nauta ${ }^{18}$ and Marsden ${ }^{19}$ have mooted suggestions that these loops, by virtue of their convergence in the striatum and pallidum, share a common modulatory mechanism which is crucial to the information-processing functions of each system. Nauta refers to the need for feedback allowing for corrective adjustments which is shared by both movement and thought, and Marsden suggests that the uniform operation carried out by the basal ganglia, 
necessary for both movement and thought, is the sequencing of the subcomponents of each. The ideas of Marsden have been articulated most fully in relation to the movement deficits seen in Parkinson's disease: he has suggested that "... the initiation and automatic execution of motor programs required to complete the motor plan of a complex motor act may depend on the basal ganglia." (p235) ${ }^{20}$ This hypothesis is particularly relevant to the present study which is concerned with planning abilities in the cognitive domain.

Several "problem solving" tasks have been described in the psychological literature but there have been few investigations of planning in relation to neurological disturbance. Shallice ${ }^{21}$ devised a simplified version of the Tower of Hanoi, renamed the Tower of London, to investigate planning abilities in groups of patients with frontal lobe lesions. An impairment in planning was found for a group of left anterior frontal lobe damaged patients but not for groups with left posterior, and right, anterior or posterior damage. For this reason, the Tower of London was the obvious choice for the present study. The task requires the subject to move coloured beads between upright poles so as to match a given pattern and the difficulty of the task can be varied in terms of the minimum number of moves to make the match. Performance is measured in terms of the number of moves required to solve the problems and also the time taken to arrive at the solutions. The structure of the task enabled its adaptation for use on a microcomputer in conjunction with a touch-sensitive screen, and this allowed for an accurate separation on the relative contributions of "thinking" and movement to the total performance times. The latencies of the Parkinson's disease group were predicted to be longer irrespective of any planning deficits, because of psychomotor impairment. Therefore, a related, yoked control condition which leads the patient through series of single moves comprising the correct solutions, was developed in order to measure motor initiation and execution times independently of planning.

The solution of a Tower of London problem involves several independent cognitive processes, some of which are spatial in nature. Subjects must first generate the sub-units which together comprise the plan, in this case the individual spatial moves. Secondly, they must organise these sub-units into a sequence which allows them to change the current pattern to the end- or goal-state. Thirdly, they must be able to maintain the derived sequence in spatial working memory as the solution is being executed. In order to assess the possible contribution of some of these component processes to any resulting deficit, two further "control" tasks were given. The two tasks chosen for this purpose also enabled further specification of the extent and nature of the visuospatial impairments which have been reported in Parkinson's disease patients. $^{2}$

The Corsi block span test ${ }^{22}$ was chosen as a simple index of a subject's ability to retain and execute a short sequence of spatial moves. This task has previously been used to demonstrate a deficit in nonverbal span in patients with right hippocampal lesions. $^{22}$ The second test controls for possible differences between the groups in aspects of spatial working memory. It is based indirectly on the Olton radial arm maze ${ }^{23}$ which requires rats to search efficiently for food in eight radial arms of a maze emanating from a central starting point. A formally similar test has also been devised for monkeys and has proved particularly sensitive to lesions in the region of the sulcus principalis area of the prefrontal cortex. ${ }^{24}$ The computerised version used here (the "Morris maze") is an adaptation of this latter task, but with added complexity to make the task sufficiently difficult for the human subject and to prevent the use of stereotyped search strategies.

The data from the Corsi span test and the maze task therefore provide important baseline information against which subjects' planning abilities can be judged. The Corsi test determines whether a subject is able to hold a sequence of spatial moves in short-term store, that is, whether they can execute a given plan of action. The higher level planning which underlies the Tower of London test requires the execution of a sequence of moves across a time period which extends beyond the usually accepted limits of any short-term store, placing demands on spatial working memory The maze test allows an assessment of this and in addition, determines whether subjects can utilise and execute the low level strategies required for efficient searching.

\section{Subjects}

The study included 12 patients with idiopathic Parkinson's disease (PD group). All patients had been diagnosed by a consultant neurologist. At the time of testing, each subject was rated for severity of symptoms using the Hoehn and Yahr scale, ${ }^{25}$ which gives a rating of their clinical disability and also classified according to whether their symptoms were predominantly right-or left-sided, or bilateral (see table 1). None of the patients had a psychiatric disorder and none were diagnosed as dementing. All patients were on levodopa

Table 1 Summary of clinical characteristics of the Parkinson's disease group

\begin{tabular}{llllllll}
\hline \multicolumn{3}{l}{ Hoehn and } & Yahr classification & \multicolumn{4}{l}{ Lateralisation } \\
\hline I & II & III & IV & V & R $>$ L & R $<$ L & R $=$ L \\
4 & 2 & 6 & 0 & 0 & 5 & 7 & 0 \\
\hline
\end{tabular}


preparations and four were, in addition, on anticholinergic drugs.

A group of 18 healthy volunteers, free from neurological or psychiatric disorder, served as controls. These were either recruited from the Cambridge area or drawn from the North East Ageing Research subject panel in Newcastle-uponTyne.

All subjects were assessed using the following battery of tests: a short form of the Wechsler Adult Intelligence Scale (WAIS) which included the Comprehension, Vocabulary, Block Design, and Object Assembly subtests; the National Adult Reading Test (NART), ${ }^{26}$ included to provide an estimate of premorbid intelligence; the Kendrick Cognitive Tests for the Elderly, ${ }^{27}$ which includes the Object Learning, and Digit Copying sub-tests.

Table 2 shows a summary of characteristics for the two groups. Student's $t$ tests confirm that the groups were matched in terms of age, $t(28)=0.525$, years of education, $t(28)=0 \cdot 266$, IQ, including both verbal, $t(28)=0.810$, and performance, $t(27)=1.984$, scores and NART estimated verbal IQ, $t(27)=0.744$. Separate $t$ tests were also run on the Block Design and Object Assembly sub-tests and on the two Kendrick tests. Various group studies have found deficits for Parkinson's disease groups in these sub-tests ${ }^{28}$ and also in tests of new learning ${ }^{29}$ and motor speed ${ }^{30}$ which comprise the Kendrick battery. The Parkinson's disease group was significantly impaired on the Object Assembly subtest, $t(27)=2 \cdot 175$, and both the Object Learning, $t(28)$ $=2.515$, and Digit Copying, $t(28)=5.012$, subtests of the Kendrick battery. None of the other comparisons were significant.

\section{Method}

All subjects were tested using an Acorn BBC + microcomputer fitted with a Microvitec colour Visual Display Unit (VDU) and touch sensitive screen (Microvitec Touch-

Table 2 Summary of characteristics of the Parkinson's disease patients and matched controls

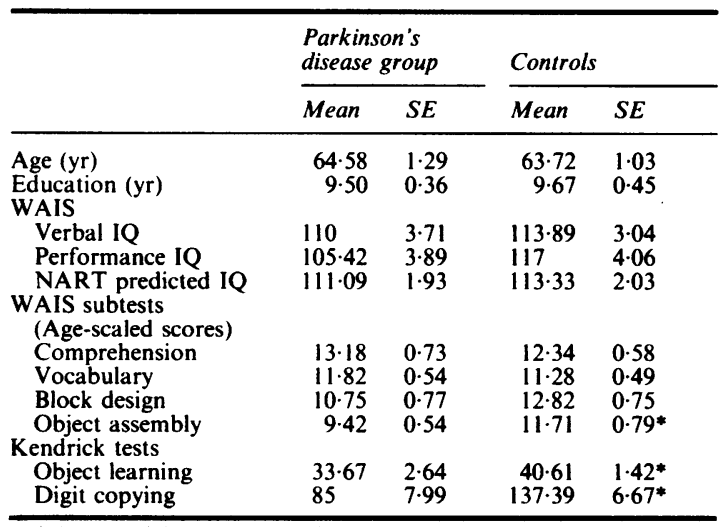

*Significant at 0.05 level (two-tailed tests) tech 501). They sat with the VDU approximately $0.5 \mathrm{~m}$ in front of them. Prior to testing, the experimenter explained that they would be required to look at the screen and make certain responses to stimuli by touching the screen. The computerised test session then began with a motor screening test which served to train the subject to point correctly but also provided useful measures of psychomotor functioning. The subjects were instructed to place the index finger of their dominant hand over the centre of a flashing cross that appeared on the VDU screen. The finger had to be held in position until 6 seconds had passed, at which point the cross moved to a new position. The test was demonstrated by the experimenter using three positions of the cross and then the subject was tested using 10 positions. This test was followed by the three experimental tests which were administered in the order given below.

\section{Computerised Corsi Block Span Test}

This test is based on the block-tapping test developed by Corsi ${ }^{22}$ as a test of spatial memory span using the sequence of paths given by Smirni et al. ${ }^{31}$ In the computerised version there is a display of nine white squares placed in the same pseudo-random arrangement as the original version. Each trial is initiated by the subject who touches a red panel at the bottom right-hand of the screen. The computer highlights a series of squares by turning them from white to blue. A square remains blue for a period of 1.5 seconds after which it returns to white and the next in sequence changes colour. On completion of the sequence, the subject has to touch the squares in the same order as they were highlighted. When a square is touched by the subject it changes colour as in the computer sequence and also emits a short tone. The subjects have to copy sequences increasing in length from one to eight. Three attempts are allowed at each level and the next level is reached if at least one sequence is copied correctly. Thus, three mistakes at any level terminates the progression.

Working Memory Test

The subject is shown an array of red squares, termed boxes, on the VDU screen. When a box is touched it opens up and reveals what is "inside". The subject is required to search through the boxes, opening them in turn until a blue "counter" is revealed. The subject then touches a white box located at the side of the screen and the object is transferred to this box. The computer then emits a short sequence of rewarding tones. Having found one counter the subject must search the remaining boxes for another counter. In other words, when a counter has been found in a box, this box will no longer have a counter in it on subsequent searches. This means that the set of boxes which potentially have counters inside them decreases as each counter is "transferred" to the side of the screen.

The subject continues searching until counters have been found in all the boxes. On each search the computer determines which box the counter will be in. Two types of error are possible: (1) Returning to a box from which a counter has already been collected on a previous search; (2) Returning to a box which has already been looked in on the same search. In this test a block is defined as a series of searches to find counters in all the boxes whilst a trial is defined as an individual search. There are four practice blocks with only two 
boxes, followed by the test blocks comprising four each of two, three, four, six, and eight box problems.

\section{Computerised Tower of London Test}

As stated above, this test is an adaptation of the human neuropsychological test developed by Shallice ${ }^{21}$ to assess planning deficits in frontal lobe patients and is based on the Tower of Hanoi problem. In the original version the subject is shown three colour-coded beads which are threaded onto upright sticks. There are three sticks of differing length in a row, the first of which can hold three beads, the second two beads and the third one bead. The subject has to move the beads to a different arrangement which is predetermined by the examiner. In the computerised version, the beads are shown in two dimensions as coloured rectangular blocks which are superimposed on upright rod structures and the set of problems devised by Shallice was used. The subject sees two arrangements on the VDU: The top one is static and represents the "goal" arrangement which the subject has to copy by moving the blocks of the bottom arrangement. Subjects were told that they had to make the bottom arrangement look like the top one, and, if possible, in a specified number of moves. Blocks could be moved by first touching the source, that is the block itself, and then the required destination. Tones were used to signify that a touch had been monitored by the computer. After the first touch, the rim of the block starts flashing and at the second touch the block moves to the new position. Subjects are not committed to a move after the first touch; by touching the same block a second time, the flashing stops and the subject can then make another choice. Illegal moves, for example attempting to move a block which was underneath another one, were explained to the subject, and if such moves were attempted, the computer gave an auditory warning signal. The program measures the number of moves that a subject takes to rearrange the blocks and provides a breakdown of the latencies for each response.

For the "yoked control" condition, the arrangements differ by just one move. As soon as the subject has made the appropriate move, the top arrangement changes so that the subject has to make another single move. For each of these single moves, the lags between stimulus presentation and the first touch, and between the first and second touches, give uncontaminated estimates of simple motor initiation and execution times respectively. The cycle of single moves continues until the subject has been led through a series of moves which corresponds exactly to those required for the correct rearrangement of blocks in the test problems. In this sense, the control trials are yoked to the test trials.

The test was designed so that the test problems are interspersed with the yoked control problems to avoid practice effects which might differentially affect performance on the two tasks. There were two sets of six test problems alternating with two sets of yoked control problems. Test problems comprised two each of 2 and 3 move problems and four each of 4 and 5 move problems, where the number of moves refers to the minimum in which solution can be achieved. The first set contained the 2,3 , and two of the 4 move problems, and the second set contained the remaining two 4 move, and the 5 move problems. Order within sets was determined by difficulty level. Prior to each set there were two practice problems, of two moves each, to ensure that the subject was following the correct rules to do the task.

\section{Results}

\section{Computerised Corsi Block Span Test}

Performance on the Corsi span test was expressed in the standard way, that is, the maximum number of squares that could be touched in correct serial order. Mean values and corresponding standard errors for the span measure were $5.00(0.26)$, and $4.58(0.19)$ for the control and Parkinson's disease groups respectively and this difference was not significant by a $t$ test, $t(28)=1 \cdot 185$. We may conclude from this that there was no deficit in spatial short-term memory capacity for the Parkinson's disease group.

\section{Working Memory Test}

The two principal measures for this test reflect the two distinct types of memory failure that could affect performance: these are denoted between-search and within-search errors. The first represents the number of returns to boxes in which counters had already been located on previous searches; the second, the number of returns to boxes which had already been looked in on that particular search.

Table 3 shows, for each of these errors, the average value for each level of difficulty (two, three, four, six, and eight boxes) of the task. More errors were made for the between- than the within-trial returns. For both, the number of errors at the two and three box difficulty levels was not much greater than zero and for the purposes of analysis only the three more difficult levels were included. A split plot 2 (group) by 3 (difficulty level) ANOVA with repeated measures on the second factor was run on each of these data sets. Within trials, errors increased significantly with number of boxes, $F(2,56)=6.219 \mathrm{p}<0.01$, but neither the group main effect, $F(1,28)=0.045$, nor the interaction, $F(2,56)=0.354$, approached significance. Thus, both groups were able to monitor very accurately the boxes searched within any particular trial. For the between-trial errors a similar pattern emerged, that is, a non-significant interaction, $F(2,56)=0.658$, and group main effect, $F(1,28)=$ $1 \cdot 037$, but a significant difficulty effect, $F(2,56)=$

Table 3 Mean errors on the working memory task

\begin{tabular}{clllll}
\hline & \multicolumn{2}{l}{ Control } & & \multicolumn{2}{l}{ Parkinson's disease group } \\
\cline { 2 - 3 } & Within & Between & & Within & \multicolumn{1}{l}{ Between } \\
\hline Block & & & & \\
2 & 0 & $0.17(0.17)$ & $0.08(0.08)$ & $0.08(0.08)$ \\
3 & 0 & $0.22(0.12)$ & 0 & $0.25(0.13)$ \\
4 & $0.39(0.24)$ & $1.61(0.65)$ & $0.75(0.46)$ & $2.33(0.93)$ \\
6 & $1.44(0.53)$ & $11.17(1.78)$ & $1.75(0.49)$ & $13.33(3.09)$ \\
8 & $5.06(2.04)$ & $27.28(3.22)$ & $3.75(1.19)$ & $32.25(3.04)$ \\
\hline
\end{tabular}

Within $=$ Within trial errors, Between $=$ Between trial errors, Standard errors, in brackets. 
Table 4 Motor initiation and execution times

\begin{tabular}{lll}
\hline & Control & Parkinson's disease group \\
\hline Initiation time (s) & $1.27(0.05)$ & $2.33(0.43)$ \\
Execution time (s) & $1.28(0.06)$ & $1.90(0.13)$ \\
\hline
\end{tabular}

Standard errors in brackets.

$111.219 \mathrm{p}<0.001$. Thus, although both groups showed an increasing susceptibility to both types of error as the number of boxes to be searched increased, the Parkinson's disease group were equivalent to controls in this respect.

\section{Computerised Tower of London Task}

Motor initiation and execution: As described above, for each problem, a separate yoked control, which led the subject through a series of single moves corresponding to a correct solution, was given. From these yoked control problems estimates of motor initiation and execution times could be derived. For this purpose, all moves were assumed to be equivalent in their motor planning demands and averages were used for the present analysis and for the derivation of thinking from total times. Table 4 shows that the mean values were as predicted, that is, the Parkinson's disease group were slower at both initiating and executing simple moves. These values were entered in a 2 (group) by 2 (movement time) ANOVA with repeated measures on the second factor. The group main effect was the only significant finding, $F(1,28)=16.070 p$ $<0.001$, thus confirming our original preduction and the findings of others. ${ }^{32}$

Total number of moves: The average number of moves for each of the four levels of difficulty is shown in table 5. If a subject failed to rearrange the blocks in the maximum number of moves, that number was used as their performance score. For each group there was a steady increase in number of moves with increasing difficulty level. Comparing these average values with the minimum number for each level, it is clear that this difference became more substantial as difficulty level increased, reflecting an increase in the relative number of failures to achieve the minimum move solution, Thus, there were very few occasions when more than the minimum number of moves was required for the first difficulty level. It is also clear that across all difficulty levels, differences between groups were only slight.

These data were used in a split plot 2 (group) by 4 (difficulty) ANOVA with groups as the between subject factor and difficulty as the within subject factor. Neither the group by difficulty interaction nor the group main effect were significant; $F(3,84)=0.378$ and $F(1,28)=0.086$ respectively. There was a significant difficulty effect, $F(3,84)=175 \cdot 676$, but given that difficulty was defined in terms of the minimum moves to solution, such a result was clearly predicted.

Latencies: The measurement of movement initiation and execution latencies in the yoked control problems meant that, for each subject, the time devoted to planning per se could be extracted from performance latencies. Two principal measures of planning time were used in analysis, each bounded by the first move: Initial planning time was the time spent formulating a set of moves prior to the first; subsequent planning time was the time subsequent to the first move during which anticipated moves are re-evaluated and new plans formulated following errors. If $\mathrm{T} 1$ is the total time prior to the first touch and T2 the time between the first touch and the end of the problem, the corresponding planning/thinking times, $\mathrm{t} 1$ and $\mathrm{t} 2$, were derived as follows: $\mathrm{t} 1=\mathrm{T} 1-\mathrm{i}, \mathrm{t} 2=\mathrm{T} 2-(\mathrm{n}-1) \mathrm{i}-\mathrm{ne}$ where $i$ and $e$ are the motor initiation and execution times respectively and $\mathrm{n}$ is the number of moves taken. In addition, each of these variables was derived from three related data sets: The first was based only on those attempts which were correct, using the minimum number of moves; the second set included all correct solutions irrespective of the number of moves; the final set averaged across all attempts, including those which did not achieve solution in the maximum number of moves.

The figure (a and b) shows the initial and subsequent planning times, derived from the complete data set, for each group as a function of difficulty. The first striking feature is that for the initial planning time there is no increase beyond that found for the three move problems, an effect seen in both groups. However, there was a steady increase across all difficulty levels for subsequent planning time.

As with the total number of moves, each data set was used in a split plot 2.4 ANOVA, with separate analyses for both the initial and subsequent planning times. From the graph it is clear that standard errors, and therefore variances, increase with mean values and this pattern was similar across the three data sets. Data were therefore log-transformed $(\log (x+1))$ in order to satisfy the assumptions underlying ANOVA.

Table 5 Average number of moves on the Tower of London test

\begin{tabular}{lll}
\hline $\begin{array}{l}\text { Difficulty level (minimum } \\
\text { number of moves) }\end{array}$ & Control & $\begin{array}{l}\text { Parkinson's disease } \\
\text { group }\end{array}$ \\
\hline 2 & $2 \cdot 06(0 \cdot 04)$ & $2^{*}$ \\
3 & $3 \cdot 58(0 \cdot 17)$ & $3.63(0 \cdot 28)$ \\
4 & $5 \cdot 74(0 \cdot 19)$ & $5 \cdot 42(0 \cdot 34)$ \\
5 & $8 \cdot 11(0 \cdot 35)$ & $8 \cdot 23(0 \cdot 73)$ \\
\hline
\end{tabular}

*All Parkinson's disease subjects solved in the minimum of moves at this level.

Standard errors in brackets. 


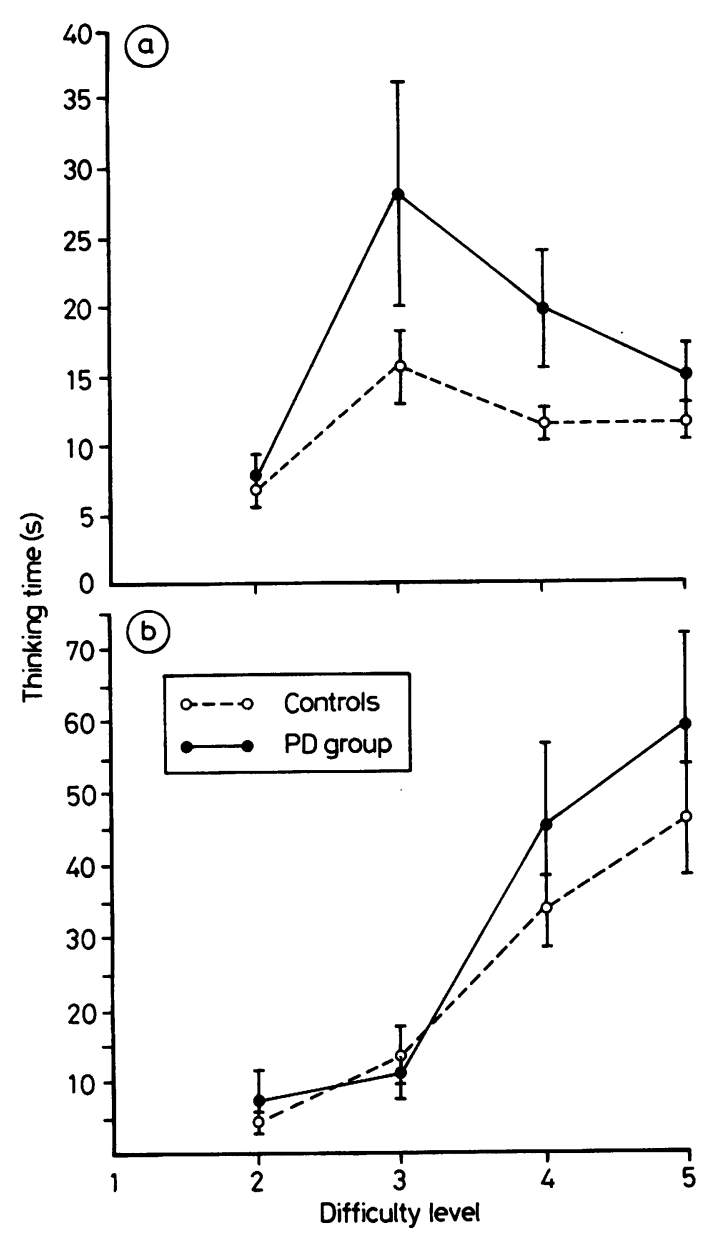

Fig Average thinking/planning times (a) prior to and (b) subsequent to the first move as a function of difficulty (minimum number of moves to solution).

Analysis of each of the data sets produced the same pattern of results: Group by difficulty interactions were not significant but difficulty was consistently significant for both initial and subsequent thinking times. However, group main effects were found only for initial thinking time, again for each data set: Correct (minimum move), $\mathrm{F}(1,28)=7.107 \mathrm{p}<0.05$; Correct (all solutions), $F(1,28)=6.300 p<0.05$; All attempts, $F(1,28)=6.277 \mathrm{p}<0.05$. Thus, overall the Parkinson's disease group took significantly longer before making the first move. Subsequent to the first move, it is apparent from fig (b) that there was a tendency for thinking times to be longer in the Parkinson's disease group but neither the interaction nor main effect was statistically significant.
Correlational analysis An important issue is whether any of the performance indices were associated with disease severity in the Parkinson's disease group. Motor initiation and execution times, initial and subsequent thinking times for each difficulty level together with the various psychometric tests were used in a correlational analysis together with Hoehn and Yahr score, illness duration, and levodopa dosage. Variation in the three disease parameters was not found to be significantly associated with any of the performance indices.

\section{Discussion}

On the Tower of London task the Parkinson's disease group solved the problems as well as controls in terms of number of moves used, but were slower in their thinking or planning time. That is, the Parkinson's disease group was as accurate but not as efficient as the control group, and the results are clear in showing a specific planning deficit in this group. The design of the task enabled the measurement of planning time independent of actual movement time, which itself was found to be impaired in the Parkinson's disease group, so the result is unconfounded in this respect.

There are two possible criticisms of this control procedure. First, the control condition clearly involves more than just the initiation and execution of a simple movement: subjects must decide how the two arrangements differ before they can formulate an appropriate motor plan. Thus, motor initiation time will be overestimated. However, for this to pose a serious threat to the interpretation of the data the control subjects should have been substantially slower in the initial processing of the stimulus arrangements, which seems unlikely. Besides, the absolute values for motor initiations would have to be considerably greater in order to account for the size of the difference between the thinking times.

Secondly, the control procedure may conversely underestimate the contribution of movement time to total performance latencies. Benecke et $a l^{33}$ have recently shown that movement execution times increase disproportionately for Parkinson's disease subjects compared with controls when discrete motor acts are linked together in a complex movement. Because movement times are estimated from single moves in the present study, and because subjects may be sequencing these single moves to form motor plans in the main condition, it follows that movement times will be underestimated and thinking/planning times overestimated, and to a greater degree for the Parkinson's disease group than for the control group. However, this possible confounding of motor and thinking latencies cannot explain the present results for the following reason. In the derivation of our $\mathrm{t} l$ 
measure a single estimate of movement initiation is used, and any underestimation of this value can be shown to be insufficient in accounting for the significant difference between the control and Parkinson's disease groups for this variable. Thus, using values given in the Benecke et al study, the average increases in movement times for the first motor act (isometric "squeeze") when performed separately and sequentially, were $-4 \%$ and $35 \%$ for the control and Parkinson's disease groups respectively. Assuming that motor initiation times for pointing are affected to a similar degree, the average difference in thinking/planning times between the two groups can be recalculated to give a value of 6.78 compared with the original $7 \cdot 13$ seconds, a trivial reduction which is unlikely to alter the conclusions.

Several other measures of cognitive performance were included mainly to serve as controls for the clarification of the precise nature of the deficit in the Parkinson's disease group. Thus, there were no group differences in the execution of a given sequence of visuospatial moves (Corsi task). Both groups were also able to generate the low level strategy required for completion of the working memory task, there being no difference in the number of within trial errors. Finally, there was no evidence of a reduction in working memory capacity for the Parkinson's disease group as there was no increase in the number of between trial errors in the same task. These factors, either alone or in combination, are potential sources of error in the performance of the Tower of London task, although the most likely outcome resulting from them would be an increase in the number of moves to solution. The lack of deficit in both short term memory spatial (that is, Corsi) span and the working memory task suggests that memory impairment was unlikely to have contributed to the observed planning deficit.

The rationale for using the Corsi span test was to establish whether Parkinson's disease subjects were able to execute a given plan of action which involved touching a set of locations in a pre-determined sequence. As noted in the introduction, a possible integrating scheme implicating "sequencing" in both the motor and cognitive deficits of Parkinson's disease has been proposed. ${ }^{20}$ More generally, there may be a problem with tasks requiring the ordering of subcomponents, for example memoranda, ${ }^{9}$ along a temporal dimension. It follows that performance on both the Corsi and the Tower of London tasks should be impaired, a prediction not supported by the present data. Further evidence that sequencing problems may be less pervasive in the motor and cognitive domains comes from Robertson and Flowers, ${ }^{34}$ who employed a task formally similar to the Corsi to investigate motor planning ability in Parkinson's disease. Sub- jects were required to perform sequences of movements in the correct order on a 5-choice button-pressing task. Parkinson's disease patients were as proficient as controls on this task suggesting, as in the present study, that the ability to remember and execute simple sequences of action is not impaired.

As far as we know, there have been no other reports of working memory in Parkinson's disease. However, the working memory task does bear some resemblance to the self-ordered pointing tasks used by Petrides and Milner, ${ }^{12}$ and found to be sensitive to frontal lobe pathology. These authors argued that their task makes considerable demands on active, working memory and deficits on it might be due to poor organisational strategies or poor monitoring of responses or both. Gotham et al $^{35}$ have reported that Parkinson's disease patients show a deficit on this self-ordered pointing task. Petrides and Milner's and our own task differ in the demands placed on working memory and perhaps also in their susceptibility to interference. As subjects work through the subjectordered tasks they must monitor variation along two dimensions, stimulus items and locations, whereas for the present task monitoring of variation along the single dimension of location is all that is required. These differences between the tasks may explain the apparently conflicting results of our own and Gotham et al's studies. It is also clear from the results of the Kendrick Object Learning Test in the present study that the Parkinson's disease group did not have completely intact memorial capacities, in agreement with the results of other studies. ${ }^{29}$

As noted in the results section, an important issue is to determine whether any significant associations exist between disease and performance indices. Patients were carefully diagnosed so as to exclude dementia and depression, and appeared to be typical in their clinical presentation of idiopathic Parkinson's disease, and all were sufficiently advanced to require medication. Gotham et $a^{35}$ have previously reported a significant positive correlation between levodopa dose and errors on a visual conditional learning task. That is, with increasing levodopa dose, performance deteriorated on this task. For the present group of Parkinson's disease patients, all correlations between disease parameters, including levodopa dose, and performance indices, were small and non-significant, whether or not those patients who were also on anticholinergic medication, were included in the analyses.

The term bradyphrenia, or psychic akinesia, has been used to describe the mental inertia or cognitive slowing which parallels the motoric form, bradykinesia, in Parkinson's disease, and several empirical demonstrations have been claimed ${ }^{936}{ }^{37}$ (but of Rafal et $a l^{38}$ ). More generally, slowing of thought processes 
has been given as a distinguishing characteristic of the sub-cortical dementias. ${ }^{39}$ The exact meaning of bradyphrenia is unclear. Slowness of thought or cognitive processing, impairment in concentration, failure of distributive attention, difficulty in making decisions, and apathy are just some of the terms that have been used. ${ }^{40}$ What seems to underlie these descriptions is the idea of a universal slowing of cognitive processes similar to that put forward to explain the diversity of changes accompanying ageing. ${ }^{41}$ Certainly, "bradyphrenic" could be used to describe the performance of the Parkinson's disease group on the Tower of London task, but this provides no insight into the precise cognitive deficit(s) necessary to produce such a reduction in speed.

Detailed psychological theories of frontal lobe function, ${ }^{2142}$ may allow a more precise characterisation of the deficit. For example, Shallice's model ${ }^{21}$ introduces the idea that the mediation of a supervisory attentional system, a general programming device necessary for satisfactory performance in nonroutine tasks such as planning, is one of the major cognitive functions subserved by the frontal lobe. A common and necessary feature of this and other theories is the need to switch or direct attention to different (memorial and perceptual) representations, ${ }^{43}$ that is, between the contents of working memory, the current act, the overall program or plan (possibly incorporating subordinate schemas, cf Shallice), and the goal state to be achieved. Our results suggest that the general programming ability Shallice speaks of, is intact in Parkinson's disease. The deficit might result from an imprecise switching mechanism which impairs the accuracy of focusing, or the selection of relevant representations with adequate resolution.

The basic difference between the present task on which a speed deficit was observed and others (for example, sorting tasks) on which accuracy is impaired is in the specification of the goal state. When present, the continual monitoring of performance is possible such that errors can be recognised and corrected: the regulation or control of behaviour by purpose or goals is normal in Parkinson's disease. For the prototypical sorting task on which impairments have been found, the goal state (that is, the answer) is of necessity not available to the subject. In the present Tower of London problems, subjects always had in view the required goal-state. Thus, depending on whether or not the goal is specified, deficits in performance which result from impaired attention switching may be evident as a reduction in either speed or accuracy. In a sense, specification of the goal state facilitates the supervision of an inefficient attention-switching device. Flowers and Robertson ${ }^{44}$ reported that Parkinson's disease patients were as accurate as controls in using a rule chosen by themselves, but when another competing alternative was introduced their performance deteriorated.

The same hypothesis can also account for the reported claims of bradyphrenia. For example, in the most recent of these, ${ }^{36}$ subjects were found to be slower on a digit symbol substitution task which requires switching attention between the symbol, a memorial representation of the code or the actual (perceptual) code, and the motor (writing) response. The purpose is clearly specified and the underlying deficit leads to a reduction in speed but no increase in errors.

The above account shares some features with other recent hypotheses of the cognitive dysfunction associated with Parkinson's disease. ${ }^{3784445}$ In a reexamination of the established set-shifting difficulties experienced by Parkinson's disease patients, Brown and Marsden ${ }^{45}$ concluded that they do not have generalised problems in switching and maintaining set, as claimed by others, but are only impaired on tasks where they have to rely upon internal control for the regulation of behaviour. As they point out, this is similar to the ideas of Taylor $\mathrm{et}^{\mathrm{al}} \mathrm{l}^{7}$ who suggest that the underlying impairment in Parkinson's disease is in "the ability to spontaneously generate efficient strategies when relying on self-directed task-specific planning". The present hypothesis suggests that it may not be the locus of control, per se, that creates problems for the Parkinson's disease group, but the strength of that control (that is, the degree to which the stimulus, or its internal representation, regulates behavioural, or computational, output). If weak and strong control lie on a continuum, then typical "internal" tasks will be found at the weak end whereas the "external" tasks used by Brown and Marsden lie at the strong end. Without strong control, the supervision of the attention-switching mechanism, hypothesised to be unreliable in Parkinson's disease, is more difficult. Thus, the number of errors on such tasks will be a function of the strength of control, but this may vary independently of the locus of control.

There is also overlap with other "switching" hypotheses, the most prominent of which is that of Cools and his colleagues ${ }^{46}$ who have suggested that Parkinson's disease patients have a diminished "shifting aptitude" that is manifested in verbal, figural, and motor modalities. Most "switching" hypotheses also converge on one further important point: they all suggest that the anatomical structure primarily responsible is the basal ganglia and this is also compatible with the present speculation. If information passing through the dysfunctioning caudate nucleus is thereby degraded, then the focus of its disinhibitory influence at the cortical level may be less precisely defined, causing "unintended" represent- 
ations to be erroneously activated.

The attention-switching hypothesis described here can account for the observed speed deficit on the Tower of London task. In addition, it may also underlie some of the deficits previously described as "bradyphrenic", and also those which have been attriuted to frontal lobe dysfunction in Parkinson's disease. The hypothesis is primarily psychological and therefore not critically dependent on the integrity, or otherwise, of specific neural structures. However, the correspondence between the present and other switching hypotheses, and their relationship to basal ganglia function, is noted.

This research was supported mainly by a Major Award from the Wellcome Trust to Drs T W Robbins, B J Everitt and S B Dunnett. Dr B J Sahakian holds an Eleanor Peel Lectureship. We are grateful to Prof P Rabbitt and Ms V Abson for allowing us access to control subjects drawn from the North East Age Research Panel at Newcastle University.

\section{References}

1 Warburton JW. Memory disturbance and the Parkinson syndrome. Br J Med Psychol 1967;40:169-71.

2 Boller F, Passafiume D, Keefe N, Rogers K, Morrow L, Kim Y. Visuospatial impairment in Parkinson's disease. Arch Neurol 1984;41:485-90

3 Lees AJ, Smith E. Cognitive deficits in the early stages of Parkinson's disease. Brain 1983;106:257-70.

4 Hietanen $M$, Teravainen $H$. Cognitive performance in early Parkinson's disease. Acta Neurol Scand 1986;73:151-9.

5 Matthews CG, Haaland KY. The effect of symptom duration on cognitive and motor performance in parkinsonism. Neurology 1979;29:951-6.

6 Brown RG, Marsden CD. How common is dementia in Parkinson's disease? Lancet 1984;ii:1262-5.

7 Taylor AE, Saint-Cyr JA, Lang AE. Frontal lobe dysfunction in Parkinson's disease. Brain 1986;109:845-83.

8 Bowen FP, Kamienny RS, Burns MM, Yahr MD. Parkinsonism: effects of levodopa treatment on concept formation. Neurology 1975;25:701-14.

9 Sagar HJ, Sullivan EV, Gabrieli JDE, Corkin S, Growden JH. Temporal ordering deficits and bradyphrenia in Parkinson's disease. Brain (in press).

10 Milner B. Some effects of frontal lobectomy in man. In: Warren JM, Akert K, eds. The Frontal Granular Cortex and Behaviour. New York: McGraw Hill, 1964:313-31.

11 Milner B. Some effects of different brain lesions on card-sorting: the role of the frontal lobes. Arch Neurol 1963;9:90-100.

12 Petrides M, Milner B. Deficits on subject-ordered tasks after frontal- and temporal-lobe lesions in man. Neuropsychologia 1982;20:249-62.

13 Milner B. Some cognitive effects of frontal lobe lesions in man. Phil Trans $R$ Soc Lond 1982;B298:211-26.

14 Scatton B, Rouqier L, Javoy-Agid F, Agid Y. Dopamine deficiency in the cerebral cortex in Parkinson's disease. Neurology 1982;32:1039-40.

15 Brozoski TJ, Brown RM, Rosvold HE, Goldman PS. Cognitive deficit caused by regional depletion of dopamine in prefrontal cortex of rhesus monkey. Science 1979;205:929-31.

16 Edwardson JA, Bloxham CA, Candy JM, Oakley AE, Perry RH, Perry EK. Alzheimers disease and Parkinson's disease: Pathol- ogical and biochemical changes associated with dementia. In: Iversen, SD, ed. Psychopharmacology: Recent Advances and Future Prospects. Oxford: Oxford University Press, 1985: $131-45$.

17 DeLong MR, Georgopoulous AP, Crutcher MD, Mitchell SJ, Richardson RT, Alexander GE. Functional organisation of the basal ganglia: contributions of single-cell recording studies. In: Functions of the Basal Ganglia. Ciba Foundation Symposium 107. London: Pitman, 1984:64-82.

18 Discussion. In: Functions of the Basal Ganglia. Ciba Foundation Symposium 107. London: Pitman, 1984:103-13.

19 Discussion. In: Functions of the Basal Ganglia. Ciba Foundation Symposium 107. London: Pitman, 1984:237-41.

20 Marsden CD. Which motor disorder in Parkinson's disease indicates the true motor function of the basal ganglia? In: Functions of the Basal Ganglia. Ciba Foundation Symposium 107. London: Pitman, 1984:225-37.

21 Shallice T. Specific impairments of planning. Phil Trans $R$ Soc Lond 1982;B298:199-209.

22 Milner B. Interhemispheric differences in the localisation of psychological processes in man. Br Med Bull 1971;27:272-7.

23 Olton DS. Spatially organised behaviours of animals: Behavioural and neurological studies. In: Potegal M, ed. Spatial Abilities. London: Pergamon, 1982.

24 Passingham RE. Memory of monkeys (Macaca mulata) with lesions in the prefrontal cortex. Behav Neurosci 1985;99:3-21.

25 Hoehn MM, Yahr MD. Parkinsonism, onset, progression and mortality. Neurology 1967;17:427-42.

26 Nelson HE. National Adult Reading Test (NART) Test Manual. Windsor: NFER-Nelson, 1982

27 Kendrick D. Cognitive Tests for the Elderly. Windsor: NFERNelson, 1985.

28 Asso D. WAIS scores in a group of Parkinson patients. $\mathrm{Br} J$ Psychiatry 1969;115:555-6.

29 Weingartner H, Burns S, Diebel R, LeWitt PA. Cognitive impairments in Parkinson's disease: Distinguishing between effort-demanding and automatic cognitive processes. Psychiatry Res 1984;11:223-35.

30 Schwab RS, England AC, Peterson E. Akinesia in Parkinson's disease. Neurology 1959;9:65-72.

31 Smirni P, Villardita C, Zappalia G. Influence of different paths on spatial memory performance in the block-tapping test. J Cl Neuropsychol 1983;3:355-9.

32 Evarts EV, Teravainen $H$, Calne DB. Reaction time in Parkinson's disease. Brain 1981;104:167-86.

33 Benecke R, Rothwell JC, Dick JPR, Day BL, Marsden CD. Disturbance of sequential movements in patients with Parkinson's disease. Brain 1987;110:361-79.

34 Robertson C, Flowers KA. Control of motor sequences in Parkinson's disease. Exp Brain Res 1987; Supp 29:S134.

35 Gotham AM, Brown RG, Marsden CD. "Frontal" cognitive functions in patients with Parkinson's disease "on" and "off" levadopa (in press).

36 Rogers D, Lees AJ, Smith E, Trimble M, Stern GM. Bradyphrenia in Parkinson's disease and psychomotor retardation in depressive illness: An experimental study. Brain 1987;110:761-76.

37 Wilson RS, Kaszniak AW, Klawans HL, Garron DC. High speed memory scanning in Parkinson's disease. Cortex 1980;16:67-72.

38 Rafal RD, Posner MI, Walker JA, Friedrich FJ. Cognition and the basal ganglia: separating mental and motor components of performance in Parkinson's disease. Brain 1984;107:1083-94.

39 Albert ML, Feldman RG, Willis AL. The 'subcortical dementia' of progressive supranuclear palsy. J Neurol Neurosurg Psychiatry 1974;37:121-30.

40 Rogers D. Bradyphrenia in Parkinson's disease: a historical review. Psychol Med 1986;16:257-65.

41 Salthouse TA, Somberg BL. Isolating the age deficit in speeded performance. J Gerontol 1982;37:59-63. 
42 Fuster JM. The prefrontal cortex, mediator of cross-temporal contingencies. Hum Neurobiol 1985;4:169-79.

43 Weber RJ, Burt DB, Noll NC. Attention switching between perception and memory. Memory and Cognition 1986;14:238-45.

44 Flowers KA, Robertson C. The effect of Parkinson's disease on the ability to maintain a mental set. J Neurol Neurosurg Psychiatry 1985;48:517-29.
45 Brown RG, Marsden CD. "Cognitive set” in Parkinson's disease. Movement Disorders (in press).

46 Cools AR, Van Den Bercken JHL, Horstink MWI, Van Spaendonck KPM, Berger HJ. Cognitive and motor shifting aptitude disorder in Parkinson's disease. J Neurol Neurosurg Psychiatry 1984;47:443-53. 